\title{
Microsurgical Varicocele Repair on Men with Grade III Lesions and Chronic Dull Scrotal Pain: A Pilot Study
}

\author{
Saad Elzanaty ${ }^{\mathrm{a}, \mathrm{c}} \quad$ Claus Erik Johansen ${ }^{\mathrm{b}}$

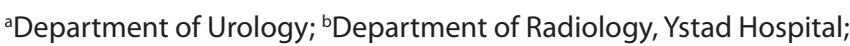 \\ 'Department of Urology, Lund University, Ystad, Sweden
}

\section{Key Words}

Microsurgery $\cdot$ Chronic scrotal pain •

Varicocele $\cdot$ Varicocele repair

\begin{abstract}
Introduction: The aim of the present study was to evaluate the effectiveness of microsurgical varicocele repair in patients with grade III lesions and chronic dull scrotal pain. Materials and Methods: The study was based on 8 patients with grade III left-sided varicocele and chronic dull scrotal pain for whom a microsurgical subinguinal varicocele repair was performed. The 1-year follow-up included pain assessment and scrotal examination. Results: Of the 8 patients, 7 (88\%) reported complete resolution of pain with no palpable varicocele on scrotal examination. No cases of testicular atrophy or hydrocele formation were reported. Conclusion: These results indicated that microsurgical varicocele repair may benefit patients with grade III lesions and chronic dull scrotal pain.

Copyright $\odot 2014$ S. Karger AG, Basel
\end{abstract}

\section{Introduction}

Varicocele defined as dilatation of the pampiniform plexus of veins surrounding the testis is observed in $10-15 \%$ of the general male population, in $20-40 \%$ of infertile men [1], and in $2-14 \%$ of men with scrotal pain $[2,3]$.

Patients with varicocele-induced male infertility present with clinical varicocele (grade I-III) and reduced semen quality. Most urologists agree that the primary

\section{KARGER}

Fax +4161306 1234

E-Mail karger@karger.ch

www.karger.com
(C) 2014 S. Karger AG, Basel

1015-9770/14/0081-0029\$38.00/0

Accessible online at:

www.karger.com/cur treatment of this group of men is varicocele repair. Patients with varicocele-induced scrotal pain present with clinical varicocele and a dull scrotal pain, generally after standing for a long period of time or performing heavy activity, and with usually an unsatisfactory response to medical treatment. Varicocele repair to manage such patients, however, is still a matter of discussion.

We report our early experience in managing patients with grade III varicocele and chronic scrotal pain with microsurgery aiming to evaluate the effectiveness of microsurgical varicocele repair in this group of patients, and at the same time to establish whether our surgical approach needs to be modified.

\section{Material and Methods}

The present study was based on 10 patients with chronic scrotal pain from 2012 to 2013. Preoperative evaluation included a detailed medical history, assessment of scrotal pain including its duration, quality (dull, dragging, or aching), and quantity using the 10-point visual analog scale for pain (VAS 0: none, 1-3: mild, 4-6: moderate, and 7-10: severe). A scrotal examination included measurement of testicular size using an orchidometer, assessment of the epididymis with respect to its consistency and any pain on palpation, and evaluation of the spermatic cord for the presence of varicocele and its location (left, right, bilateral). Varicoceles were assigned to grades I-III during examination while the patients were in a standing position according to the criteria of Lyon et al. [5] as follow: grade I: palpable only with Valsalva, grade II: palpable without Valsalva, and grade III: visible from distance. Finally, the patients were examined for signs of inguinal hernia.

Patients who had other causes of scrotal pain such as testicular trauma, testicular torsion, epididymitis, prostatitis, sexually transmitted diseases, and inguinal hernia were excluded. Only patients with clinical varicocele and chronic scrotal pain were included 
Table 1. Descriptive statistics of the study population

\begin{tabular}{ll}
\hline Variables & Value \\
\hline Age (years) & $21(18-24)$ \\
VAS & $4-10$ \\
Varicocele grade & 0 \\
I & 0 \\
II & 8 \\
III & \\
Varicocele laterality & 8 \\
Unilateral & 0 \\
Bilateral & \\
\hline
\end{tabular}

and were scheduled for microsurgical subinguinal varicocele repair. All the patients underwent medical treatment for pain with non-steroidal anti-inflammatory medications (6-12 months), and were given instruction about scrotal elevation and limitation of heavy activity. None of the patients derived any significant benefit from the medical treatment.

Varicocele repair was performed using the microsurgical subinguinal approach with the patients under general anaesthesia by 1 surgeon (S.E.Z.). A $2-3 \mathrm{~cm}$ transverse skin incision overlying the external inguinal ring was done. The incision was extended through the Camper's and Scarpa's fascia, allowing the spermatic cord to be grasped with a Babcock clamp. Special care was always taken to identify and spare the ilioinguinal nerve. No testicular delivery was done. Any external spermatic veins were isolated and divided. The spermatic cord was viewed at a magnification from $\times 3$ to $\times 16$ using a microscope (OMPI ORL S5; Carl Zeiss) with hand control focusing, zooming, and a 1-head unit. The external and internal spermatic fascias were incised and the vas deferens and its vasculature were separated. A Doppler probe was used to identify the testicular artery when it was difficult to identify with the operating microscope. Care was taken to preserve the lymphatics in order to prevent the development of hydrocele. Internal spermatic veins were then clipped, ligated, and divided. The patients were then observed in the postoperative department and were discharged the same day.

Follow-up evaluation was done at 1-month (for early postoperative complications: wound infection and hematoma), and at 1-year after surgery (for late postoperative complications: testicular atrophy, hydrocele formation, and persistence/recurrence of varicocele), and assessment of scrotal pain using the VAS. Follow-up evaluation was done by 1 examiner. A written consent from each patient was obtained.

Patient's responses after surgery were graded as complete response (pain was absent after surgery), partial response (pain persisted but was reduced after surgery), and no response (pain remained unchanged after surgery). Surgical success was defined as absence of palpable varicocele on scrotal examination, and a report of scores in the lowest range of the VAS $(\leq 1$ point).

\section{Results}

The study was based on a total of 10 patients with chronic scrotal pain. Two patients were found to have chronic epididymitis (one on the left side and one on the
Table 2. Results of microsurgical varicocele repair on 8 men with grade III lesions and chronic dull pain

\begin{tabular}{ll}
\hline Variables & Patient, $\mathrm{n}$ \\
\hline Pain resolution (VAS) & \\
$\quad$ Complete & $7(0-1)$ \\
Partial & $1(4)$ \\
Postoperative complications & \\
Wound infection & 0 \\
Hematoma & 0 \\
Hydrocele & 0 \\
Testicular atrophy & 0 \\
Persistence/recurrence & 1 \\
\hline
\end{tabular}

right side). The criteria for this diagnosis were as follow: previous history of epididymitis including medical treatment, painful epididymis, and no varicocele on clinical examination. These 2 men were excluded from the study. Scrotal examination of the remaining 8 patients revealed normal testicular size, normal epididymal findings, and grade III left-sided varicocele. None of the patients had any signs of inguinal hernia. All patients reported a dull non-radiating scrotal pain that increased in intensity with exercise. The pain was of more than 6 months duration. The quantity of pain using the VAS pain scale ranged from 4 to 10 . The mean age of the men was 21 years (range 18-24 years)(Table 1).

Following varicocele repair, the 8 patients attended the initial postoperative visit at the 1-month follow-up and 6 did so at the 1-year follow-up. The remaining 2 patients showed up 1 month later after many attempts were made to contact them.

The mean operative time was 65 minutes (range 47-78 minutes). No intraoperative or early postoperative complications such as wound infection or scrotal hematoma were reported. At 1-year follow-up, 7 of the $8(88 \%)$ patients reported complete response (VAS, 0 ), with no palpable varicocele on scrotal examination. Only 1 patient $(12 \%)$ reported partial scrotal pain (VAS, 4). On scrotal examination, he had grade II varicocele on the left side (the operation side), and had gained grade II varicocele on the right side. No evidence of testicular atrophy or hydrocele formation was found in any patient (Table 2).

\section{Discussion}

Based on this study, 8 patients with grade III leftsided varicocele and chronic dull scrotal pain underwent microsurgical subinguinal varicocele repair and were followed-up for 1 year after surgery with assessment of pain, and scrotal examination. Of these 8 patients, 7 $(88 \%)$ reported complete resolution of pain. Our results are in accordance with previous reports $[2,4,6,7]$. We 
believe, therefore, that microsurgical varicocele repair should be considered in men with grade III lesions and chronic dull pain.

The success of surgical treatment of painful varicocele has been reported to depend on the character of the pain. Thus, it has been observed that patients who presented with dull pain had a significantly higher rate of success in terms of resolution of pain than those who presented with sharp pain [3]. Also, the duration of pain was reported to affect the success of surgical treatment of painful varicocele. In this regard, men who presented with a long period of pain ( $>3$ months) were associated with a significantly higher rate of success in terms of resolution of pain after surgery as compared to men who presented with a short period of pain ( $<3$ months) [6]. In accordance, our patients presented with dull non-radiating chronic scrotal pain.

Another factor that plays an important role in the success of surgical treatment of painful varicocele is its persistence/recurrence. In this matter, microsurgical varicocele repair was reported to be associated with a significantly lower rate of persistence/recurrence than non-microsurgical varicocele repair $[8,9]$. The contribution of the gubernacular vein to postoperative persistence/recurrence is still unclear. Goldstein et al. [10] performed 33 varicocele repairs using the non-microsurgical approach without delivery of the testis, 12 varicocele repairs using $2.5 \times$ loupes without delivery of the testis, and 326 varicocele repairs using the microsurgical approach with delivery of the testis and reported a persistence/recurrence rate of 9,8 , and $0.6 \%$, respectively. Schiff et al. [11] performed 74 varicocele repairs using the microsurgical subinguinal approach. In each of the 74 procedures, the testis was delivered and the gubernaculum was examined for the presence of any varicose veins between the gubernacula and the testis and any large veins were clipped and ligated. They reported no persistence/recurrence during the follow-up period which was extended to a mean of 10 months. These results suggest that delivery of the testis and examination of the gubernacular vein reduce the risk of persistence/recurrence. The patients included in this study underwent varicoceles repair using the microsurgical subinguinal approach. However, we did not deliver the testis out of the scrotum nor did we ligate the gubernacular vein, and persistence/recurrence was found in 1 patient. We intend to run another study similar to the present one with delivery of the testis and examination of the gubernacular veins and compare its results with the results from the present study.

The resolution of scrotal pain after surgery could be attributed to the reduction of the pressure in the testicular venous drainage system, thus enabling normal oxygen flow to the testicles with correction of hypoxia in the testicular tissue caused by hydrostatic pressure resulting from the destruction of the 1-way valves in internal spermatic veins. In addition, resection of some of the spermatic nerves during surgery is another contributing factor for pain resolution.

With the assistance of the operating microscope and the Doppler probe, we were able to identify and preserve the testicular artery and the testicular lymphatics. None of our patients had testicular atrophy or hydrocele 1 year after surgery.

This was a small study and the study population represents a selected group of patients with a specific type of painful varicocele and very selective entry criteria. However, the results are still comparable to those from previous reports.

In conclusion, the results from this study indicate that microsurgical varicocele repair may benefit patients with grade III lesions and chronic dull scrotal pain.

\section{References}

1 Meacham RB, Townsend RR, Rademacher $\mathrm{D}$, Drose JA: The incidence of varicoceles in the general population when evaluated by physical examination, gray scale sonography and color Doppler sonography. J Urol 1994;151:1535-1538.

2 Peterson AC, Lance RS, Ruiz HE: Outcomes of varicocele ligation done for pain. J Urol 1998;159:1565-1567.

3 Al-Buheissi SZ, Patel HR, Wazait HD, Miller RA, Nathan S: Predictors of success in surgical ligation of painful varicocele. Urol Int 2007;79:33-36.

4 Yaman O, Ozdiler E, Anafarta K, Gogus O: Effect of microsurgical subinguinal varicocele ligation to treat pain. Urology 2000; 55:107-108.
5 Lyon RP, Marshall S, Scott MP: Varicocele in childhood and adolescence: implication in adulthood infertility? Urology 1982;19:641644.

6 Altunoluk B, Soylemez H, Efe E, Malkoc O: Duration of preoperative scrotal pain may predict the success of microsurgical varicocelectomy. Int Braz J Urol 2010;36:55-59.

7 Kim SO, Jung H, Park K: Outcomes of microsurgical subinguinal varicocelectomy for painful varicoceles. J Androl 2012;33:872875 .

8 Cayan S, Kadioglu TC, Tefekli A, Kadioglu A, Tellaloglu S: Comparison of results and complications of high ligation surgery and microsurgical high inguinal varicocelectomy in the treatment of varicocele. Urology 2000;55:750-754.
9 Diegidio P, Jhaveri JK, Ghannam S, Pinkhasov R, Shabsigh R, Fisch H: Review of current varicocelectomy techniques and their outcomes. BJU Int 2011;108:1157-1172.

10 Goldstein M, Gilbert BR, Dicker AP, Dwosh $\mathrm{J}$, Gnecco C: Microsurgical inguinal varicocelectomy with delivery of the testis: an artery and lymphatic sparing technique. J Urol 1992;148:1808-1811.

11 Schiff J, Kelly C, Goldstein M, Schlegel P, Poppas D: Managing varicoceles in children: results with microsurgical varicocelectomy. BJU Int 2005;95:399-402. 